\title{
Characterizing Computed Tomography-Detected Arterial Hyperenhancing-Only Lesions in Patients at Risk of Hepatocellular Carcinoma: Can Non-Contrast Magnetic Resonance Imaging Be Used for Sequential Imaging?
}

\author{
So Hyun Park, $M D^{1}$, Bohyun Kim, $M D^{2}$, So Yeon Kim, $M D^{3}$, Seung Joon Choi, $M D^{1}$, \\ Jimi Huh, $M D^{2}$, Hye Jin Kim, $M D^{2}$, Kyung Won Kim, $M D^{3}$, Seung Soo Lee, $M D^{3}$ \\ ${ }^{1}$ Department of Radiology, Gil Medical Center, Gachon University College of Medicine, Incheon, Korea; ${ }^{2}$ Department of Radiology, Ajou University \\ Hospital, Ajou University School of Medicine, Suwon, Korea; ${ }^{3}$ Department of Radiology and Research Institute of Radiology, Asan Medical Center, \\ University of Ulsan College of Medicine, Seoul, Korea
}

Objective: To test the feasibility of non-contrast magnetic resonance imaging (MRI) in a sequential imaging study for characterizing computed tomography (CT)-detected arterial-enhancing nodules that do not washout in patients at risk of hepatocellular carcinoma (HCC).

Materials and Methods: In this retrospective study, 134 patients (mean age \pm standard deviation, $56.8 \pm 10.0$ years) with 151 arterial enhancing-only nodules measuring up to $2 \mathrm{~cm}$ during multiphasic CT that were subsequently evaluated using gadoxetic acid-enhanced MRI in treatment-naïve at-risk patients from three tertiary referral centers were included. Tentative diagnostic criteria for HCC and hepatic malignancy were defined as the presence of one of eight MRI features favoring HCC in combinations of the following sequences: T2-weighted imaging (T2WI), diffusion-weighted imaging (DWI), T1-weighted dual gradient-echo in-phase and out-of-phase imaging (Dual-GRE), and hepatobiliary phase imaging (HBP). Typical hemangiomas and arterioportal shunts were excluded from the analysis. Diagnostic performance for HCC and hepatic malignancy was calculated and compared between the abbreviated MRI and full-sequence gadoxetic acid-enhanced MRI.

Results: Of 151 nodules (mean size, $1.2 \mathrm{~cm}$ ) $68 \mathrm{HCCs}$ and 83 non-HCC benignities and malignancies were included. The combination of T2WI, DWI, and Dual-GRE showed per-lesion sensitivity, specificity, and accuracy of $88.2 \%, 90.4 \%$, and $89.4 \%$, respectively, comparable to those of full-sequence MRI. Applying the same sequence combination to diagnose hepatic malignancy had per-lesion sensitivity, specificity, and accuracy of $86.8 \%, 97.3 \%$, and $92.1 \%$. In nodules $<1 \mathrm{~cm}$, adding HBP increased sensitivity by up to $13 \%$ without compromising the specificity or accuracy.

Conclusion: The non-contrast MRI protocol comprising T2WI, DWI, and Dual-GRE showed reasonable and comparable performance to full-sequence MRI for discriminating HCC and primary liver malignancies in CT-detected indeterminate arterial enhancingonly nodules in at-risk patients, and can be potentially used for sequential imaging in place of a full-sequence MRI. In nodules $<1 \mathrm{~cm}, \mathrm{HBP}$ may still be needed to preserve sensitivity.

Keywords: Hepatocellular carcinoma; Liver; Magnetic resonance imaging; Noncontrast MRI

Received June 25, 2019; accepted after revision November 4, 2019.

This study was supported by a research fund from the Korean Society of Radiology through Radiology Imaging Network of Korea for Clinical Research (RINK-CR) and the Scientific Research Fund of the Korean Liver Cancer Study Group.

Corresponding author: Bohyun Kim, MD, Department of Radiology, Ajou University Hospital, Ajou University School of Medicine, 164 World cup-ro, Yeongtong-gu, Suwon 16499, Korea.

- Tel: (8231) 219-7094•Fax: (8231) 219-5862•E-mail: kbh@ajou.ac.kr

This is an Open Access article distributed under the terms of the Creative Commons Attribution Non-Commercial License (https:// creativecommons.org/licenses/by-nc/4.0) which permits unrestricted non-commercial use, distribution, and reproduction in any medium, provided the original work is properly cited. 


\section{INTRODUCTION}

Hepatocellular carcinoma (HCC) is one of the few cancers that can be diagnosed solely using noninvasive imaging studies. Major contemporary guidelines proposed by the American Association for Study of Liver Diseases, Liver Imaging Reporting and Data System (LI-RADS), European Association for the Study of the Liver, Asian Pacific Association for the Study of the Liver, Korean Liver Cancer Association-National Cancer Center (KLCANCC), and Japanese Society of Hepatology all allow confirmative diagnosis of HCC by identifying the hallmarks of the tumor, i.e., arterial phase hyperenhancement (APHE) and portal venous or delayed phase washout, on multiphasic CT and MRI using extracellular or liver-specific contrast agents (1-6). The diagnostic algorithms of these guidelines recommend performing multiphasic CT or MRI to establish a diagnosis of HCC in nodules discovered during surveillance, typically with ultrasound, while CT and MRI are also endorsed in Eastern guidelines for certain groups of patients. Should these nodules lack hallmarks of HCC and thus are deemed indeterminate, various options, including follow-up imaging, a second round of imaging with an alternative modality or contrast agent, or biopsy, are possible for further characterization (1-3).

Among indeterminate nodules, arterial-enhancing nodules that do not washout are often encountered on multiphasic $\mathrm{CT}$ in cirrhotic livers and impose a diagnostic challenge. In liver cirrhosis, hepatic parenchymal distortion and blood flow redistribution result in arterial-enhancing lesions that are not HCC, particularly arterioportal (AP) shunts (7). Because up to $60 \%$ of small HCCs may not demonstrate washout $(8,9)$, such arterial enhancing-only nodules require sequential diagnostic imaging either immediately or in follow-up (1-3). Although there is yet no consensus suggesting which imaging modality should be used for this purpose, many centers in Eastern countries prefer gadoxetic acid (Eovist or Primovist; Bayer HealthCare, Berlin, Germany)-enhanced MRI because of its high sensitivity in hepatobiliary phase imaging (HBP) (10-12). However, this technique is expensive, and because dynamic imaging has already been obtained from multiphasic CT, it is unclear whether gadoxetic acid-enhanced MRI needs additional dynamic imaging. With this, we hypothesized that adding a non-contrast MRI protocol omitting dynamic imaging and HBP may have adequate diagnostic value. If noncontrast MRI with shortened sequences could be used to characterize indeterminate hypervascular lesions detected on multiphasic CT in at-risk patients, the need to perform full-sequence MRI may be reduced. Therefore, this study aimed to determine the feasibility of non-contrast MRI for characterizing nodules with arterial hypervascularity alone detected on multiphasic CT in patients at risk of HCC.

\section{MATERIALS AND METHODS}

This retrospective study was approved by the Institutional Review Board of three institutions (Ajou University Hospital, Asan Medical Center, and Gil Medical (enter), and the requirement for written informed consent was waived. The present report constitutes a substudy based on data from a multicenter study that has been registered on the domestic trial registry of the Republic of Korea (cris.nih. go.kr: KCT0003221).

\section{Patient Selection}

We retrospectively searched 844 consecutive patients with chronic liver disease who underwent gadoxetic acid-enhanced MRI to evaluate indeterminate, arterialenhancing nodules detected during multiphasic CT surveillance less than 3 months apart while attending the liver unit at Ajou University Hospital and Gil Medical Center between January 2015 and March 2017, and at Asan Medical Center between January 2013 and December 2013. A further selection identified 670 nodules in 591 patients according to the following inclusion criteria: 1 ) discrete round or ovoid nodules with APHE on multiphase CT without prior treatment for HCC; 2) nodules with no or equivocal washout in the portal venous and delayed phases ("equivocal washout" was defined as any discrepancy in the interpretation of washout appearance between two readers during the later formal image review); 3 ) nodules $0.5-2.0 \mathrm{~cm}$ in diameter numbering three or less per patient; and 4) nodules that were not typical hemangiomas (nodules with marked T2 hyperintensity equivalent to the intensity of cerebrospinal fluid and the usual peripheral globular, progressive centripetal enhancement pattern) or AP shunts (subcapsular lesions with wedge-shaped arterial enhancement of straight border without washout) $(4,7)$. If there were four or more suspicious nodules in the patient, we included only the three largest nodules. We excluded 348 patients who had been treated using transcatheter arterial chemoembolization or radiofrequency ablation and thus lacked a pathological diagnosis, as well as 149 
patients with insufficient follow-up (<18 months). Finally, 151 nodules in 134 patients were included (Fig. 1). The nodules were selected and annotated by study coordinators from each center (all with more than 7 years of experience in hepatic imaging) who were not involved in the image analysis. Of these nodules, 33 nodules had been included in a previous study (13).

\section{Imaging Protocols}

\section{CT Techniques}

All CT examinations were performed using various multidetector CT scanners (SOMATOM Sensation 64, SOMATOM Definition, or SOMATOM Definition Flash; Siemens Healthineers, Forchheim, Germany). Acquisition protocols varied between centers, with a reconstruction thickness between $2 \mathrm{~mm}$ and $5 \mathrm{~mm}$. All multiphasic CT examinations comprised unenhanced, late hepatic arterial (30-35 seconds), portal venous (75-90 seconds), and delayed (3 minutes) phases. In total, $1.5-2 \mathrm{~mL} / \mathrm{kg}$ of iodine contrast media, with an iodine concentration of $300 \mathrm{~g} / \mathrm{L}$, was injected using a power injector at a rate of $3-4 \mathrm{~mL} / \mathrm{s}$, not exceeding $150 \mathrm{~mL}$ in total volume.

\section{MRI Techniques}

MRI examinations were performed using 1.5T and 3T scanners at Asan Medical Center (Magnetom Avanto 1.5T and Skyra 3T; Siemens Healthineers), 3T scanners at Gil Medical Center (Verio 3T and Skyra 3T; Siemens Healthineers), and 1.5T and $3 \mathrm{~T}$ scanners at Ajou University Hospital (Signa HDx 1.5T and Discovery MR750w 3T; GE Healthcare, Milwaukee, MI, USA). HBP was obtained 15-20 minutes after a bolus injection of $0.025 \mathrm{mmol} / \mathrm{kg}(0.1 \mathrm{~mL} /$ $\mathrm{kg}$ ) gadoxetic acid.

\section{Image Analysis}

CT and MR images were anonymized, randomized, and independently reviewed by two board-certified abdominal radiologists, each with more than 7 years of experience in hepatic imaging. Images from each reviewer's own center were reviewed on-site and those from other centers were remotely accessed. The largest diameter and location of each nodule according to the Couinaud classification were recorded. In four MRI sequences of the sequential MRI examination, the presence of the following eight imaging features that favor a diagnosis of $\mathrm{HCC}$ were evaluated in each nodule $(12,14-16)$ : 1) mild-to-moderate hyperintensity on T2-weighted imaging (T2WI); 2) hyperintensity on diffusion weighted imaging (DWI) (b-value

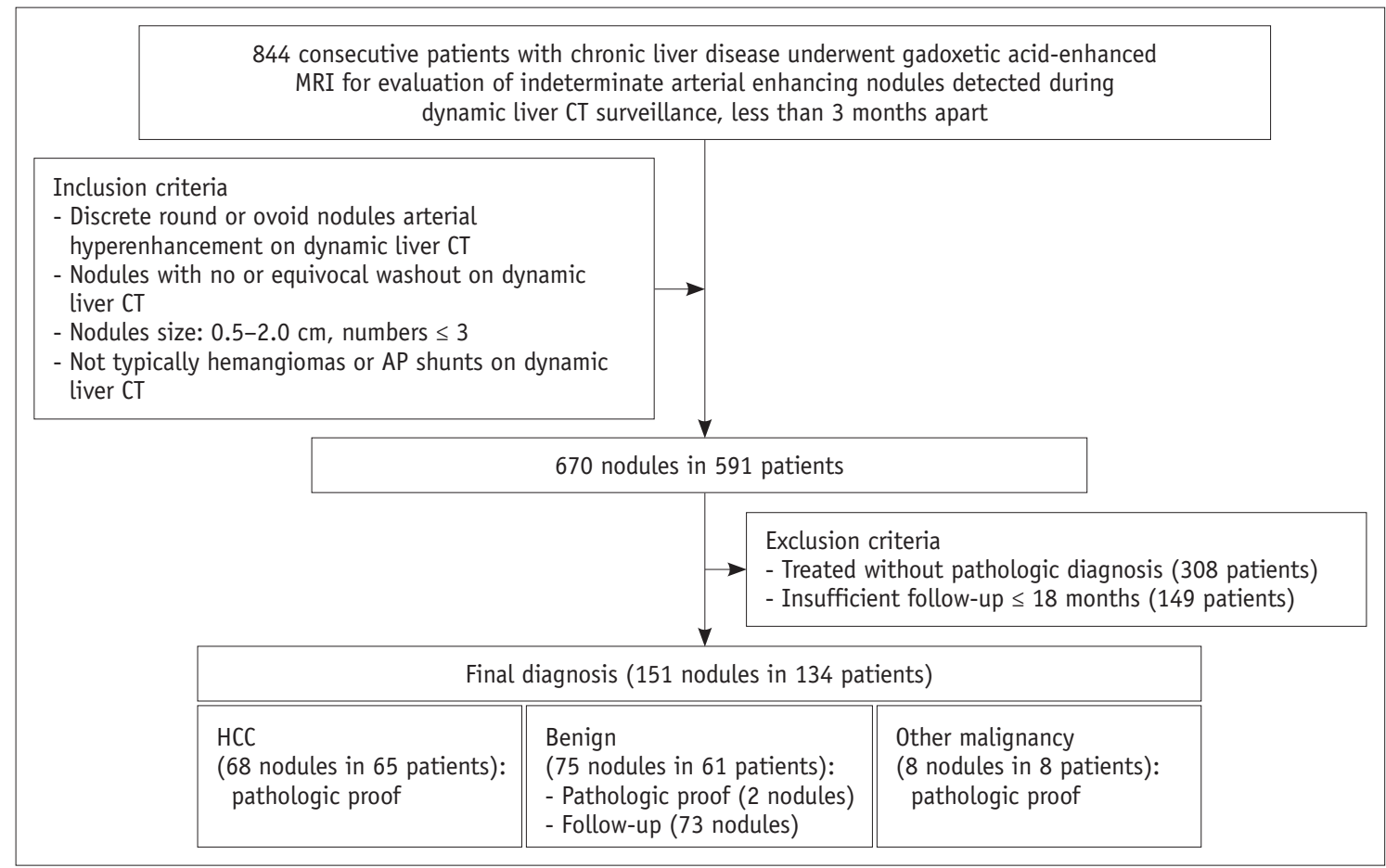

Fig. 1. Patient flow diagram. $\mathrm{AP}=$ arterioportal, $\mathrm{CT}=$ computed tomography, $\mathrm{HCC}=$ hepatocellular carcinoma, $\mathrm{MRI}=$ magnetic resonance imaging 
of $400-500$ or $\left.800 \mathrm{~s} / \mathrm{mm}^{2}\right)$; 3) hypointensity on HBP; 4) fat content on T1-weighted dual gradient-echo in-phase and out-of-phase imaging (Dual-GRE); 5) non-enhancing capsule; 6) hemorrhagic content; 7) nodule-in-nodule; and 8) mosaic appearance. Regarding the last four features, the sequence in which the feature was observed was specified for each nodule. Any between-reader discrepancies concerning the presence or absence of imaging features were resolved by consensus discussion.

The diagnosis of HCC was suspected when at least one of the eight imaging features was identified on T2WI, DWI, HBP, or Dual-GRE. Nodules with none of these features were considered as benign entities and their tentative diagnoses were given. To further explore the diagnostic performance for hepatic malignancy, the same provisional radiologic criteria for HCC was applied to indicate hepatic malignancy.

The nodules were then re-reviewed after two weeks on a full-sequence gadoxetic acid-enhanced MRI including dynamic enhancement, three non-contrast MRI sequences (T2WI, DWI, and Dual-GRE), and HBP by the same independent readers to diagnose HCC. The diagnosis was made when the arterial hyperenhancing-only nodules on MRI showed one of the eight imaging features described above, the nodules demonstrated the hallmarks of HCC (APHE and portal venous washout), or the nodules showed only portal venous washout $(1,2,4)$.

\section{Statistical Analysis}

Clinical characteristics were compared across patients with HCCS and non-HCCs (benign lesions and other malignant tumors) using a one-way analysis of variance with a post hoc Tukey's test (for continuous variables) or the $\chi^{2}$ test (for categorical variables). To determine the abbreviated MRI sequence most predictive of HCC, perlesion sensitivity, specificity, and accuracy of the four MRI sequences alone or in combination with others were calculated and compared to each other and to those of the full-sequence gadoxetic acid-enhanced MRI using generalized estimating equations. In a subgroup analysis, per-lesion analyses were performed according to the size of the nodules $(1-2 \mathrm{~cm}$, or $<1 \mathrm{~cm})$. Diagnostic performance for hepatic malignancy was also evaluated by calculating the per-lesion sensitivity, specificity, and accuracy. A $p$ value $<0.05$ was considered statistically significant. All statistical analyses were performed using SPSS Statistics for Windows, Version 21.0 (IBM Corp., Armonk, NY, USA) and R software, Version 3.5.1 (The R Foundation for Statistical
Computing, Vienna, Austria).

\section{RESULTS}

\section{Patient Characteristics and Pathologic Diagnosis of Nodules}

There were 99 men (74\%) and 35 women (26\%) with a mean age \pm standard deviation of $56.8 \pm 10.0$ years (range, $30-82$ years) in the study. Hepatitis $B$ was the predominant cause of chronic liver disease $(n=115 ; 85.8 \%)$, followed by hepatitis $\mathrm{C}$ and alcoholic hepatitis. The clinical characteristics of 134 patients are summarized in Table 1.

of 151 nodules (mean size, $1.2 \mathrm{~cm}$; range, $0.5-2 \mathrm{~cm}$ ), 78 nodules from 75 patients were histopathologically diagnosed by either hepatic resection $(n=75)$, or coreneedle biopsy $(n=3)$ to include $68 \mathrm{HCCs}$, eight combined hepatocellular cholangiocarcinoma (CHCC-CC), one dysplastic nodule (DN), and one focal nodular hyperplasia (FNH). Of them, $13 \mathrm{HCCS}$ and one DN showed equivocal washout (9.3\%). The remaining 73 benign lesions, including $69 \mathrm{AP}$ shunts, two DNs, and two FNHs, were diagnosed based on typical imaging features and stability during a follow-up period of at least 18 months $(7,17)$. There was a significant difference in the mean size of the nodules between patients with and without $\mathrm{HCC}(p<0.05)$.

\section{Prevalence of MRI Features in HCC and Benign Entities}

The prevalence of the eight MRI features in HCC and benign lesions according to their size are shown in Table 2. In the $68 \mathrm{HCCs}$, diffusion restriction $(n=62 ; 91.2 \%)$, hypointensity on $\operatorname{HBP}(n=63 ; 92.6 \%)$, and mild-tomoderate hyperintensity on T2WI $(n=59 ; 86.8 \%)$ were the three most common imaging features followed by fat deposition ( $n=11 ; 16.2 \%)$. All eight MRI features were significantly more common in $\mathrm{HCC}$ (all $p<0.05$ ).

\section{Diagnostic Performance of Abbreviated MRI for HCC}

The per-lesion diagnostic performance of abbreviated MRI for HCC is shown in Table 3 and Supplementary Table 1. Among various combinations of four MRI sequences, the combination of T2WI, DWI, and Dual-GRE (Figs. 2, 3) for detecting two or more MRI features showed acceptably high accuracy (89.4\%), specificity $(90.4 \%)$, and sensitivity $(88.2 \%)$. Conversely, the combination of all four sequences resulted in the lowest specificity $(84.3 \%)$ but slightly higher sensitivity (91.2\%). There was no significant difference in the sensitivity, specificity, or accuracy 
Table 1. Clinical Characteristics of Study Patients

\begin{tabular}{|c|c|c|c|c|c|}
\hline \multirow{2}{*}{ Characteristics } & \multirow{2}{*}{$\begin{array}{c}\text { All Patients } \\
\text { (151 Nodules) }\end{array}$} & \multirow{2}{*}{$\begin{array}{c}\text { Patients with } \mathrm{HCC} \\
\text { (68 Nodules) }\end{array}$} & \multicolumn{2}{|c|}{ Patients with Non-HCC $(n=83)$} & \multirow{2}{*}{$P$} \\
\hline & & & Benign (75 Nodules) & Malignant (8 Nodules) & \\
\hline No. of patients & 134 & 65 & 61 & 8 & \\
\hline Age (years) & $56.8 \pm 10.0$ & $57.5 \pm 9.4$ & $55.6 \pm 10.9$ & $52.5 \pm 6.5$ & 0.395 \\
\hline Sex (male/female) & $99 / 35$ & $44 / 21$ & $51 / 10$ & $4 / 4$ & 0.036 \\
\hline Mean nodule size $(\mathrm{cm})$ & $1.2 \pm 0.3$ & $1.3 \pm 0.3$ & $1.1 \pm 0.3$ & $1.4 \pm 0.2$ & $<0.001^{*}$ \\
\hline Causes of cirrhosis & & & & & 0.200 \\
\hline Hepatitis B & $115(85.8)$ & $60(92.3)$ & $47(77.0)$ & $8(100)$ & \\
\hline Hepatitis C & $7(5.2)$ & $1(1.5)$ & $6(9.8)$ & $0(0)$ & \\
\hline Hepatitis B, C & $1(0.7)$ & $1(1.5)$ & $0(0)$ & $0(0)$ & \\
\hline Alcoholic liver disease & $7(5.2)$ & $1(1.5)$ & $6(9.8)$ & $0(0)$ & \\
\hline Other & $4(3.0)$ & $2(3.1)$ & $2(3.3)$ & $0(0)$ & \\
\hline Child-Pugh classification & & & & & 0.144 \\
\hline A & $118(88.1)$ & $60(92.3)$ & $51(83.6)$ & $7(87.5)$ & \\
\hline B & $13(9.7)$ & $2(3.1)$ & $10(16.4)$ & $1(12.5)$ & \\
\hline C & $3(2.2)$ & $3(4.6)$ & $0(0)$ & $0(0)$ & \\
\hline \multicolumn{6}{|l|}{ Tumor marker value } \\
\hline $\operatorname{AFP}(\mathrm{ng} / \mathrm{mL})$ & $48.7 \pm 158.2$ & $69.4 \pm 202.7$ & $31.7 \pm 103.3$ & $17.8 \pm 21.4$ & 0.334 \\
\hline PIVKA-II & $31.9 \pm 36.1$ & $32.4 \pm 37.4$ & $27.1 \pm 17.6$ & $41.6 \pm 59.8$ & 0.628 \\
\hline
\end{tabular}

Otherwise specified, data in parentheses are percentage. *Significant difference between HCC and benign lesion. AFP $=$ alpha fetoprotein, $\mathrm{HCC}=$ hepatocellular carcinoma, PIVKA-II = protein-induced by vitamin $\mathrm{K}$ absence or antagonist-II

Table 2. Prevalence of Various Imaging Features of CT-Detected Indeterminate Arterial Enhancing Nodules according to Pathology and Size

\begin{tabular}{|c|c|c|c|c|c|c|c|}
\hline \multirow[b]{2}{*}{ Imaging Features } & \multicolumn{3}{|c|}{$\mathrm{HCC}$} & \multicolumn{3}{|c|}{ Benign Lesions } & \multirow[b]{2}{*}{$P$} \\
\hline & $\begin{array}{l}<1 \mathrm{~cm} \\
(\mathrm{n}=8)\end{array}$ & $\begin{array}{c}1-2 \mathrm{~cm} \\
(\mathrm{n}=60)\end{array}$ & $\begin{array}{c}\text { All } \\
(n=68)\end{array}$ & $\begin{array}{c}<1 \mathrm{~cm} \\
(\mathrm{n}=28)\end{array}$ & $\begin{array}{l}1-2 \mathrm{~cm} \\
(\mathrm{n}=47)\end{array}$ & $\begin{array}{c}\text { All } \\
(n=75)\end{array}$ & \\
\hline Diffusion restriction & $6(75.0)$ & $56(93.3)$ & $62(91.2)$ & $0(0)$ & $3(6.3)$ & $3(4.0)$ & $<0.001$ \\
\hline Moderate high T2 signal & $6(75.0)$ & $53(88.3)$ & $59(86.8)$ & $0(0)$ & $5(10.6)$ & $5(6.7)$ & $<0.001$ \\
\hline Fat deposition & $2(25.0)$ & $9(15.0)$ & $11(16.2)$ & $0(0)$ & $1(2.1)$ & $1(1.3)$ & 0.001 \\
\hline Non-enhancing capsule & $0(0)$ & $2(3.3)$ & $2(2.9)$ & $0(0)$ & $1(2.1)$ & $1(1.3)$ & 0.012 \\
\hline Nodule in nodule appearance & $0(0)$ & $1(1.7)$ & $1(1.5)$ & $0(0)$ & $0(0)$ & $0(0)$ & 0.019 \\
\hline Mosaic appearance & $0(0)$ & $1(1.7)$ & $1(1.5)$ & $0(0)$ & $0(0)$ & $0(0)$ & 0.019 \\
\hline Blood product & $0(0)$ & $1(1.7)$ & $1(1.5)$ & $0(0)$ & $0(0)$ & $0(0)$ & 0.019 \\
\hline Hepatobiliary phase hypointensity & $7(87.5)$ & $56(93.3)$ & $63(92.6)$ & $0(0)$ & $4(8.5)$ & $4(5.3)$ & $<0.001$ \\
\hline
\end{tabular}

Data represent number of nodules, while those in parentheses are percentages.

between combinations (all $p>0.05$ ). Of note, in the subgroup analysis, sensitivities were lower in HCCs smaller than $1 \mathrm{~cm}$ across all sequences except for the combination of HBP and Dual-GRE.

In full-sequence gadoxetic acid-enhanced MRI, per-lesion sensitivity, specificity, and accuracy for HCC diagnosis were $92.7 \%, 86.8 \%$, and $89.4 \%$, respectively, comparable to those of non-contrast MRI with or without HBP (all $p>0.05$ ). On dynamic enhancement, of 68 HCCs, 36 (52.9\%) showed hallmarks of HCC, 25 (36.8\%) showed APHE only, and two $(2.9 \%)$ showed washout only. Considering the equivalent diagnostic performance, non-contrast MRI composed of
T2WI, DWI, and Dual-GRE could be the best-performing sequential imaging study, comparable to a full-sequence MRI.

Eight $\mathrm{CHCC}-\mathrm{CC}$, three AP shunts, two DNs, and two FNHs ranging $1-1.3 \mathrm{~cm}$ were falsely diagnosed as HCC. All cHCCCCs showed mild to moderate hyperintensity on T2WI, diffusion restriction, and hypointensity on $\mathrm{HBP}$, indicating HCC per the predefined diagnostic criteria. Two DNs, one AP shunt, and one FNH showed hypointensity on HBP, and the remaining two AP shunts and one FNH demonstrated mild to moderate hyperintensity on T2WI. In terms of falsenegative diagnoses, three HCCs ranging 1-1.1 cm failed 
Table 3. Per-Lesion Diagnostic Performance of Abbreviated MRI Sequence(s) for HCC

\begin{tabular}{|c|c|c|c|c|c|c|}
\hline Diagnostic Criteria & Sensitivity (\%)* & $P^{\dagger}$ & Specificity (\%)* & $P^{\dagger}$ & Accuracy $(\%)^{*}$ & $P^{\dagger}$ \\
\hline \multicolumn{7}{|l|}{ One or more findings" } \\
\hline T2WI & 86.8 [76.4-94.7] & $0.347^{\ddagger}$ & $84.3[74.7-91.4]$ & All $>0.990^{\ddagger}$ & $86.0[79.4-91.1]$ & All $>0.910^{\ddagger}$ \\
\hline DWI & $91.2[81.8-96.7]$ & $0.407^{\ddagger}$ & $88.0[79.0-94.1]$ & & $89.4[83.4-93.8]$ & \\
\hline HBP & 92.7 [83.7-97.6] & $0.701^{\ddagger}$ & $85.5[76.1-92.3]$ & & $88.7[82.6-93.3]$ & \\
\hline T2WI, Dual-GRE & 89.7 [79.9-95.8] & $0.550^{\ddagger}$ & $84.3[74.7-91.4]$ & & 86.8 [80.3-91.7] & \\
\hline DWI, Dual-GRE & $94.1[85.6-98.4]$ & $0.756^{\ddagger}$ & $86.8[77.5-93.2]$ & & 90.1 [84.2-94.3] & \\
\hline HBP, Dual-GRE & $94.1[85.6-98.4]$ & & $85.5[76.1-92.3]$ & & $89.4[83.4-93.8]$ & \\
\hline \multicolumn{7}{|l|}{ Two or more findings } \\
\hline T2WI, HBP & 86.8 [76.4-93.8] & $0.609^{\S}$ & $88.0[79.0-94.1]$ & All $>0.970^{\S}$ & $87.4[81.1-92.3]$ & All $>0.980^{\S}$ \\
\hline DWI, HBP & 89.7 [79.9-95.8] & $0.774^{\S}$ & $89.2[80.4-94.9]$ & & $89.4[83.4-93.8]$ & \\
\hline T2WI, DWI & $85.3[74.6-92.7]$ & $0.512^{\S}$ & $90.4[81.9-95.8]$ & & $88.1[81.8-92.8]$ & \\
\hline T2WI, DWI, Dual-GRE & $88.2[78.1-94.8]$ & $0.744^{\S}$ & $90.4[81.9-95.8]$ & & $89.4[83.4-93.8]$ & \\
\hline T2WI, DWI, Dual-GRE, HBP & $91.2[81.8-96.7]$ & & $84.3[74.7-91.4]$ & & 87.4 [81.1-92.3] & \\
\hline $\begin{array}{l}\text { Full-sequence gadoxetic } \\
\text { acid-enhanced MRI }\end{array}$ & 92.7 [83.7-97.6] & $>0.990^{q, * *}$ & $86.8[77.5-93.2]$ & $>0.990^{q 1, * *}$ & $89.4[83.4-93.8]$ & $>0.990 * *$ \\
\hline
\end{tabular}

Number in square brackets are $95 \%$ confidence intervals. *Percentage out of 151 nodules, ${ }^{\dagger}$ Bonferroni-correct $p$ values, ${ }^{\ddagger}$ Compared to combination of HBP and Dual-GRE, ${ }^{8}$ Compared to combination of all four sequences, "Phrase "One or more findings" refers to presence of more than one finding among eight imaging features, known to favor diagnosis of HCC, "Compared to combination of T2WI, DWI, and Dual-GRE, ${ }^{*}$ Compared to combination of T2WI, DWI, Dual-GRE, and HBP. Dual-GRE = dual gradient-echo in-phase and out-of-phase imaging, DWI = diffusion-weighted imaging, $\mathrm{HBP}=$ hepatobiliary phase imaging, $\mathrm{T} 2 \mathrm{WI}=\mathrm{T} 2$-weighted imaging

to demonstrate any of the eight MRI features and were subsequently not diagnosed as HCC. Upon review of their full-sequence MRI studies, dynamic enhancement using gadoxetic acid did not demonstrate washout either on portal venous phase or transitional phase, leading them to be missed even in the full-sequence MRI.

\section{Diagnostic Performance of Abbreviated MRI for Hepatic Malignancy}

The per-lesion performance of abbreviated MRI in diagnosing hepatic malignancy is shown in Table 4 and Supplementary Table 2. Combining Dual-GRE with HBP resulted in the highest accuracy $(94.7 \%)$, sensitivity $(94.7 \%)$, and specificity $(94.7 \%)$ in the detection of at least one MRI feature deemed to indicate hepatic malignancy. To detect two or more MRI features, the combination of T2WI, DWI, and Dual-GRE resulted in a sensitivity, specificity, and accuracy of $86.8 \%, 97.3 \%$, and $92.1 \%$, respectively. There was no significant difference in the sensitivity, specificity, or accuracy between sequences (all $p>0.05$ ).

\section{DISCUSSION}

Our study demonstrated that sequential non-contrast MRI comprising T2WI, DWI, and Dual-GRE has reasonable and comparable performance to full-sequence gadoxetic acid- enhanced MRI for diagnosing HCC and hepatic malignancies in indeterminate arterial-enhancing nodules smaller than $2 \mathrm{~cm}$ detected on multiphasic CT in patients at risk of HCC. With this, non-contrast MRI could be used as a sequential radiological examination to characterize arterial hypervascular nodules that require further assessment.

The best-performing combination of non-contrast sequences had comparable sensitivity, specificity, and accuracy to combinations added with HBP and to those of full-sequence gadoxetic acid-enhanced MRI in current and previous studies, reporting sensitivity, specificity, and accuracy of $76.0-91.9 \%, 81.2-93.6 \%$, and $82.7 \%$, respectively $(14,18-20)$. Of note, on dynamic enhancement MRI, $36(52.9 \%)$ of 68 HCCs showed APHE and portal venous washout, while two $(2.9 \%)$ showed portal venous washout only. These results indicate that additional dynamic enhancement MRI could reveal hallmarks of HCC in some of the indeterminate nodules. However, given the comparable diagnostic performance of non-contrast MRI, our suggested diagnostic scheme still may play a potential role as sequential imaging after multiphasic CT in place of full-sequence MRI.

We used provisional diagnostic criteria of $\mathrm{HCC}$ and showed their future validity as formal criteria for multiphasic CT integrated with abbreviated MRI. Using LI-RADS V2018, and assuming that the nodules lacked threshold growth, 


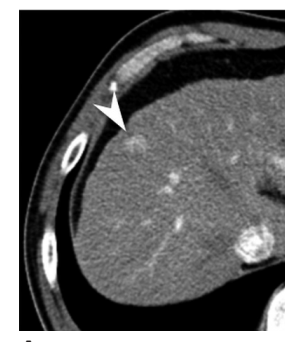

A

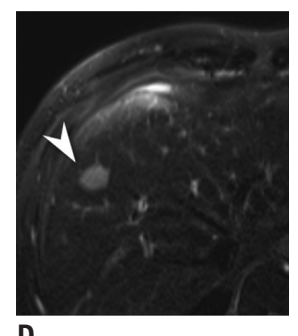

D

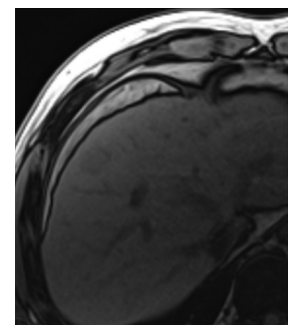

$\mathbf{G}$

Fig. 2. HCC in 55-year-old man with chronic hepatitis B.

Multiphasic CT revealed $1.8-\mathrm{cm}$ arterial enhancing nodule (arrowhead) in segment 8 of liver (A), with no washout in portal venous phase

(B) or in 3-minute delayed phase (C). On T2-weighted image, nodule (arrowhead) shows mildly high signal intensity (D), with diffusion restrictions (arrowheads) $(\mathbf{E}, \mathbf{F})\left(\mathrm{b}=800 \mathrm{~s} / \mathrm{mm}^{2}\right)$ and without any fat component on T1-weighted dual gradient-echo images $(\mathbf{G}, \mathbf{H})$. This nodule was correctly diagnosed as HCC by combining non-contrast MRI.
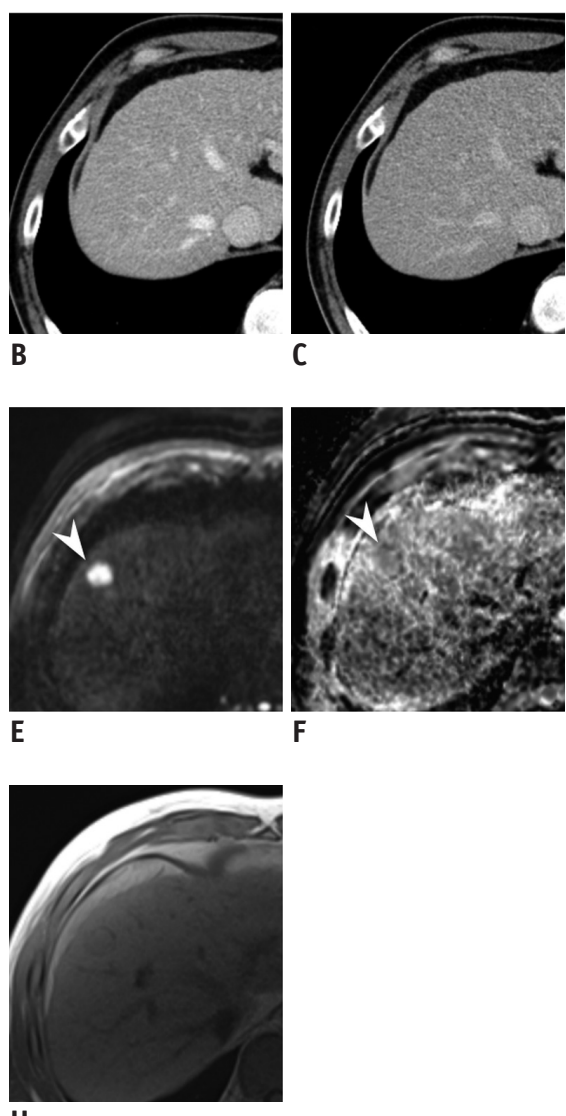

$\mathrm{H}$

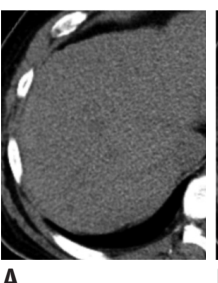

A
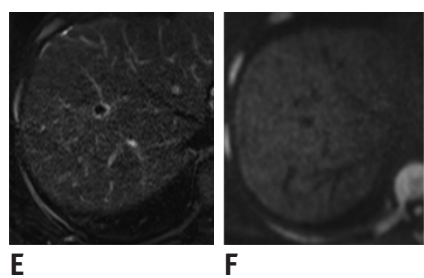

$\mathbf{F}$
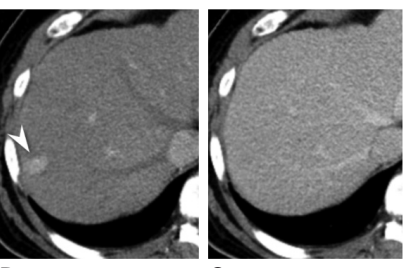

C
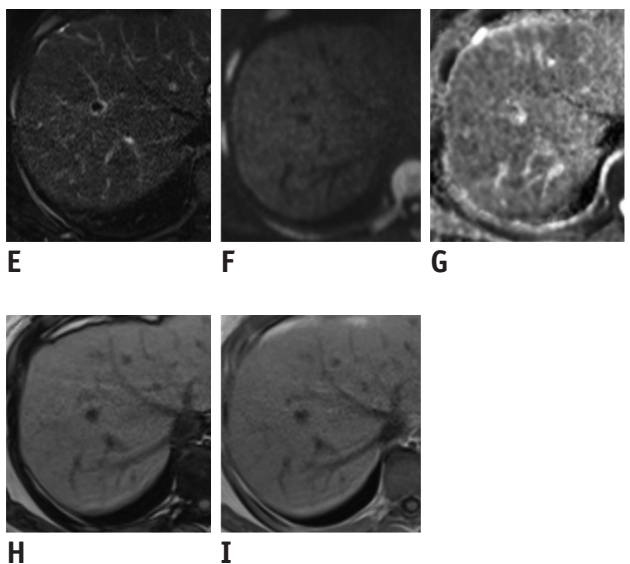

Fig. 3. Arterioportal shunt in 48-year-old man with chronic hepatitis $B$.

Multiphasic $\mathrm{CT}$ revealed $1.9-\mathrm{cm}$ arterial enhancing nodule in segment 8 of liver (arrowhead) (A, B), with no washout in portal venous phase (C) or in 3-minute delayed phase (D). There is no high signal intensity in matching area on T2-weighted image $(\mathbf{E})$, no diffusion restriction $(\mathbf{F}, \mathbf{G})$ $\left(b=800 \mathrm{~s} / \mathrm{mm}^{2}\right)$, and no fat component on T1-weighted dual gradientecho images (H, I). The nodule was correctly diagnosed as non-HCC by combining non-contrast MRI.

Table 4. Per-Lesion Diagnostic Performance of Abbreviated MRI Sequence(s) for Hepatic Malignancy

\begin{tabular}{|c|c|c|c|c|c|c|}
\hline Diagnostic Criteria & Sensitivity $(\%)^{*}$ & $P^{\dagger}$ & Specificity $(\%)^{*}$ & $P^{\dagger}$ & Accuracy $(\%)^{*}$ & $P^{\dagger}$ \\
\hline \multicolumn{7}{|l|}{ One or more findings } \\
\hline T2WI & 86.8 [77.1-93.5] & $0.296^{\ddagger}$ & $92.0[83.4-97.0]$ & All $>0.950^{\ddagger}$ & 89.4 [83.4-93.8] & $0.470^{\ddagger}$ \\
\hline DWI & 90.8 [81.9-88.8] & $0.359^{\ddagger}$ & 96.0 [88.8-99.2] & & 93.4 [88.2-96.8] & $0.595^{\ddagger}$ \\
\hline $\mathrm{HBP}$ & 93.4 [85.3-97.8] & $0.709^{\ddagger}$ & 94.7 [86.9-98.5] & & 94.0 [89.0-97.2] & $0.835^{\ddagger}$ \\
\hline T2WI, Dual-GRE & 89.5 [80.3-95.3] & 0.434 & $92.0[83.4-97.0]$ & & 90.7 [84.9-94.8] & 0.592 \\
\hline DWI, Dual-GRE & 93.4 [85.3-97.8] & $0.508^{\ddagger}$ & 94.7 [86.9-98.5] & & 94.0 [89.0-97.2] & $0.768^{\ddagger}$ \\
\hline HBP, Dual-GRE & $94.7[87.1-98.6]$ & & $94.7[86.9-98.5]$ & & 94.7 [89.8-97.7] & \\
\hline \multicolumn{7}{|l|}{ Two or more findings } \\
\hline T2WI, HBP & 86.8 [77.1-93.5] & $0.544^{\S}$ & 96.0 [88.8-99.2] & All $>0.970^{\S}$ & 91.4 [85.7-95.3] & $0.721^{\S}$ \\
\hline DWI, HBP & 89.5 [80.3-95.3] & $0.723^{\S}$ & 97.3 [90.7-99.7] & & 93.4 [88.2-96.8] & $0.942^{\S}$ \\
\hline T2WI, DWI & $84.2[74.0-91.6]$ & $0.458^{\S}$ & 97.4 [90.7-99.7] & & 90.7 [84.9-94.8] & $0.657^{\S}$ \\
\hline T2WI, DWI, Dual-GRE & 86.8 [77.1-93.5] & $0.610^{\S}$ & 97.3 [90.7-99.7] & & 92.1 [86.5-95.8] & $0.831^{\S}$ \\
\hline T2WI, DWI, Dual-GRE, HBP & $92.1[83.6-97.1]$ & & 93.3 [85.1-97.8] & & 92.7 [87.3-96.3] & \\
\hline
\end{tabular}

Numbers in square brackets are $95 \%$ confidence intervals. *Percentage out of 151 nodules, ${ }^{\dagger}$ Bonferroni-correct $p$ values, ${ }^{\ddagger}$ Compared to combination of HBP and Dual-GRE, 'Compared to combination of all four sequences, "Phrase "One or more findings" refers to presence of more than one finding among eight imaging features, known to favor diagnosis of HCC. 
the majority of HCCs in our study would be categorized as "probably HCC (LR-4)" but never "definite HCC (LR-5)" because ancillary features cannot upgrade an observation to definite HCC (4). The updated KLCA-NCC guideline opened a potential diagnostic pathway for abbreviated MRI by introducing "probable HCC" to specify nodules having some of the hallmarks of HCC and at least one of the ancillary features (5). Although the definition mostly fits our diagnostic criteria, it still refers to "probable HCC" instead of "definite $\mathrm{HCC}$ ", incongruous with the results of the current study. Further study could establish the diagnostic criteria for abbreviated MRI alone or in combination with multiphasic CT.

In studies assessing the performance of gadoxetic acidenhanced MRI in HCC diagnosis, HBP improved sensitivity at the cost of specificity $(14,21,22)$. Similarly, HBP alone or in combination with other sequences increased the sensitivity by $3 \%$, while decreasing the specificity up to $6 \%$ compared to the combination of all three non-contrast sequences in overall nodules, though without statistical significance. Specificity-wise, of 15 nodules falsely diagnosed as HCCs, 12 nodules showed hypointensity on HBP either with or without T2 hyperintensity or diffusion restriction. Considering the cost and medical resources required for $\mathrm{HBP}$, non-contrast MRI may be a feasible sequential examination that could reduce the healthcare budget while preserving the diagnostic performance necessary for $\mathrm{HCC}$. However, in nodules smaller than $1 \mathrm{~cm}$, HBP increased the sensitivity by approximately $13 \%$ without compromising the specificity. Gadoxetic acid significantly improves the sensitivity for small $(<2 \mathrm{~cm}) \mathrm{HCCs}$ because it has a high lesion-to-background contrast ratio on HBP $(21,23,24)$. Similar benefits may have contributed to the remarkable boost in sensitivity for HCC in smaller nodules. Thus, HBP might still be necessary when characterizing CTdetected nodules smaller than $1 \mathrm{~cm}$.

In our study, eight CHCC-CCs were falsely diagnosed as HCC by both readers, majorly jeopardizing the specificity for HCC diagnosis, corroborated by the $7-8 \%$ increase in specificity for diagnosing hepatic malignancy that includes both HCC and CHCC-CC. Notably, one of the readers interpreted two such nodules as showing rim APHE, which is considered a feature suggesting non-HCC malignancy (LR-M) according to LI-RADS v2018 (4). Current LI-RADS does not permit integrated interpretation across modalities and thus assigning LI-RADS categories to our proposed combination of multiphasic CT and abbreviated non-contrast MRI is not valid for now (25). However, selectively adopting features suggesting non-HCC entities would likely increase the specificity for HCC diagnosis.

The present study has several limitations. First, it was a retrospective study, which has an inherent selection bias, and a prospective study is required for validation of the results; based on our exclusion criteria, many nodules were exempted from the final analysis, which may have led to a greater selection bias. Specifically, AP shunts were excluded based on their typical appearance on CT. However, because we intended to reflect actual clinical practice, which usually disregards typical AP shunts but pays full attention to atypical nodular AP shunts not to misdiagnose HCCs, we believe such an exclusion is justified. Additionally, many benign nodules including two DNs were diagnosed and included based on imaging features and stability over follow-up. To fully adopt non-contrast MRI as a sequential imaging, a prospective study including populations with and without a history of HCC is needed. Second, the performance of adding non-contrast MRI was not directly compared with those of other imaging methods, including multiphasic MRI using extracellular gadolinium contrast agent. Third, CT and MRI parameters varied between centers and that may have affected the diagnostic performance. Furthermore, the tissue signal of the liver on Dual-GRE and non-contrast T1-weighted image may be inconsistent across different field strengths (26). However, this is an innate limitation of multicenter, cross-platform studies. Finally, we did not evaluate other incidental lesions discovered on reviewing MRI sequences. Hence, we may have underestimated the performance of HBP. However, it was beyond the scope of this study to evaluate such lesions.

In conclusion, non-contrast MRI comprising T2WI, DWI, and Dual-GRE could be sequentially used to distinguish HCC in CT-detected indeterminate arterial-enhancing nodules in at-risk patients and can be potentially used for sequential imaging in place of a full-sequence MRI. In nodules smaller than $1 \mathrm{~cm}$, HBP may still be needed to preserve the sensitivity.

\section{Supplementary Materials}

The Data Supplement is available with this article at https://doi.org/10.3348/kjr.2019.0447.

\section{Conflicts of Interest}

The authors have no potential conflicts of interest to 
disclose.

\section{Acknowledgments}

The statistical analysis of the study was provided by the Office of Biostatistics, Ajou Research Institute for Innovation Medicine, Ajou University Medical Center, Suwon, Korea.

\section{ORCID iDs}

Bohyun Kim

https://orcid.org/0000-0003-1157-415X

So Hyun Park

https://orcid.org/0000-0001-9935-2863

So Yeon Kim

https://orcid.org/0000-0001-6853-8577

Seung Joon Choi

https://orcid.org/0000-0003-3861-7682

Jimi Huh

https://orcid.org/0000-0002-8832-6165

Hye Jin Kim

https://orcid.org/0000-0002-0263-1581

Kyung Won Kim

https://orcid.org/0000-0002-1532-5970

Seung Soo Lee

https://orcid.org/0000-0002-5518-2249

\section{REFERENCES}

1. Heimbach JK, Kulik LM, Finn RS, Sirlin CB, Abecassis MM, Roberts LR, et al. AASLD guidelines for the treatment of hepatocellular carcinoma. Hepatology 2018;67:358-380

2. European Association for the Study of the Liver. EASL clinical practice guidelines: management of hepatocellular carcinoma. J Hepatol 2018;69:182-236

3. Omata M, Cheng AL, Kokudo N, Kudo M, Lee JM, Jia J, et al. Asia-Pacific clinical practice guidelines on the management of hepatocellular carcinoma: a 2017 update. Hepatol Int 2017;11:317-370

4. CT/MRI LI-RADS ${ }^{\circledR}$ v2018 CORE. American College of Radiology, 2018. Available at: https://www.acr.org/-/media/ACR/Files/ RADS/LI-RADS/LI-RADS-2018-Core.pdf?la=en. Accessed June 20, 2019

5. Korean Liver Cancer Association (KLCA) and National Cancer Center (NCC). 2018 Korean Liver Cancer Association-National Cancer Center Korea practice guidelines for the management of hepatocellular carcinoma. Korean J Radiol 2019;20:10421113

6. Kudo M, Izumi N, Kokudo N, Matsui 0, Sakamoto M, Nakashima 0 , et al. Management of hepatocellular carcinoma in Japan: consensus-based clinical practice guidelines proposed by the
Japan Society of Hepatology (JSH) 2010 updated version. Dig Dis 2011;29:339-364

7. Choi BI, Lee KH, Han JK, Lee JM. Hepatic arterioportal shunts: dynamic CT and MR features. Korean J Radiol 2002;3:1-15

8. Sherman M. Diagnosis of small hepatocellular carcinoma. Hepatology 2005;42:14-16

9. Aubé C, Oberti F, Lonjon J, Pageaux G, Seror 0, N'Kontchou G, et al. EASL and AASLD recommendations for the diagnosis of HCC to the test of daily practice. Liver Int 2017;37:1515-1525

10. Kim SH, Kim SH, Lee J, Kim MJ, Jeon YH, Park Y, et al. Gadoxetic acid-enhanced MRI versus triple-phase MDCT for the preoperative detection of hepatocellular carcinoma. AJR Am J Roentgenol 2009;192:1675-1681

11. Liu X, Jiang H, Chen J, Zhou Y, Huang Z, Song B. Gadoxetic acid disodium-enhanced magnetic resonance imaging outperformed multidetector computed tomography in diagnosing small hepatocellular carcinoma: a meta-analysis. Liver Transpl 2017;23:1505-1518

12. Motosugi U, Ichikawa T, Sou H, Sano K, Tominaga L, Muhi A, et al. Distinguishing hypervascular pseudolesions of the liver from hypervascular hepatocellular carcinomas with gadoxetic acid-enhanced MR imaging. Radiology 2010;256:151-158

13. Park SH, Kim B, Kim SY, Shim YS, Kim JH, Huh J, et al. Abbreviated MRI with optional multiphasic CT as an alternative to full-sequence MRI: LI-RADS validation in a HCC-screening cohort. Eur Radiol 2019 Dec 19 [Epub ahead of print]. https://doi.org/10.1007/s00330-019-06546-5

14. Choi SH, Byun JH, Lim YS, Yu E, Lee SJ, Kim SY, et al. Diagnostic criteria for hepatocellular carcinoma $\leq 3 \mathrm{~cm}$ with hepatocyte-specific contrast-enhanced magnetic resonance imaging. J Hepatol 2016;64:1099-1107

15. Kim TK, Lee KH, Jang HJ, Haider MA, Jacks LM, Menezes RJ, et al. Analysis of gadobenate dimeglumine-enhanced MR findings for characterizing small $(1-2-\mathrm{cm})$ hepatic nodules in patients at high risk for hepatocellular carcinoma. Radiology 2011;259:730-738

16. Piana G, Trinquart L, Meskine N, Barrau V, Beers BV, Vilgrain V. New MR imaging criteria with a diffusion-weighted sequence for the diagnosis of hepatocellular carcinoma in chronic liver diseases. J Hepatol 2011;55:126-132

17. Campos JT, Sirlin CB, Choi JY. Focal hepatic lesions in Gd-EOB-DTPA enhanced MRI: the atlas. Insights Imaging 2012;3:451-474

18. Duncan JK, Ma N, Vreugdenburg TD, Cameron AL, Maddern G. Gadoxetic acid-enhanced MRI for the characterization of hepatocellular carcinoma: a systematic review and metaanalysis. J Magn Reson Imaging 2017;45:281-290

19. Kim SY, An J, Lim YS, Han S, Lee JY, Byun JH, et al. MRI with liver-specific contrast for surveillance of patients with cirrhosis at high risk of hepatocellular carcinoma. JAMA Oncol 2017;3:456-463

20. Roberts LR, Sirlin CB, Zaiem F, Almasri J, Prokop LJ, Heimbach JK, et al. Imaging for the diagnosis of hepatocellular 
carcinoma: a systematic review and meta-analysis. Hepatology 2018;67:401-421

21. Choi JY, Lee JM, Sirlin CB. CT and MR imaging diagnosis and staging of hepatocellular carcinoma: part II. Extracellular agents, hepatobiliary agents, and ancillary imaging features. Radiology 2014;273:30-50

22. Joo I, Lee JM, Lee DH, Jeon JH, Han JK, Choi BI. Noninvasive diagnosis of hepatocellular carcinoma on gadoxetic acidenhanced MRI: can hypointensity on the hepatobiliary phase be used as an alternative to washout? Eur Radiol 2015;25:2859-2868

23. Kierans AS, Kang SK, Rosenkrantz AB. The diagnostic performance of dynamic contrast-enhanced MR imaging for detection of small hepatocellular carcinoma measuring up to $2 \mathrm{~cm}$ : a meta-analysis. Radiology 2016;278:82-94

24. Ahn SS, Kim MJ, Lim JS, Hong HS, Chung YE, Choi JY. Added value of gadoxetic acid-enhanced hepatobiliary phase MR imaging in the diagnosis of hepatocellular carcinoma. Radiology 2010;255:459-466

25. Sirlin C. Use of the liver imaging reporting and data system in hepatocellular carcinoma. Gastroenterol Hepatol (N Y) 2017;13:363-365

26. Chang KJ, Kamel IR, Macura KJ, Bluemke DA. 3.0T MR imaging of the abdomen: comparison with $1.5 \mathrm{~T}$. Radiographics 2008;28:1983-1998 